\title{
Cadherin-17 is a useful diagnostic marker for adenocarcinomas of the digestive system
}

\author{
Min-Cheng $\mathrm{Su}^{1}$, Ray-Hwang Yuan ${ }^{2}$, Chiao-Ying Lin $^{3}$ and Yung-Ming Jeng ${ }^{4}$ \\ ${ }^{1}$ Department of Pathology, Min-Sheng General Hospital, Taoyuan City, Taiwan, ROC; ${ }^{2}$ Department of Surgery, \\ National Taiwan University Hospital, Taipei, Taiwan, ROC; ${ }^{3}$ Department of Dentistry, College of Medicine, \\ National Taiwan University, Taipei, Taiwan, ROC and ${ }^{4}$ Department of Pathology, National Taiwan University \\ Hospital, and Department of Pathology, College of Medicine, National Taiwan University, Taipei, Taiwan, \\ $R O C$
}

\begin{abstract}
Cadherin-17, also called liver-intestine cadherin, is a calcium-dependent transmembrane glycoprotein that mediates cell-cell adhesion in intestinal epithelium. Expression of cadherin-17 was reported in gastric, pancreatic, and colorectal adenocarcinomas but not in other tumors. Whether cadherin-17 can be used as a marker for diagnosis of cancers is still unclear. In this study, we used immunohistochemical methods to stain cadherin-17 in tissue arrays containing most normal tissues and 518 carcinomas from many anatomic sites. Among normal tissues, the expression of cadherin-17 was limited to epithelial cells of small intestine and colon. Colorectal adenocarcinomas showed staining in $96 \%$ of cases and most of them had strong and diffuse staining. Gastric, pancreatic, and biliary adenocarcinomas showed diffuse or scattered staining in about $25-50 \%$ of cases. Fewer than $1 \%$ of carcinomas outside the digestive system were positive for cadherin-17. When a two-marker, Cadherin-17/cytokeratin 7, profile was used, 37 of $38(97 \%)$ cadherin-17( + )/cytokeratin $7(-)$ tumors were colorectal adenocarcinomas; 49 of $56(86 \%)$ cadherin-17(+)/cytokeratin $7(+)$ tumors were gastric, pancreatic, or biliary adenocarcinomas. Our results show that cadherin-17 is a useful immunohistochemical marker for diagnosis of adenocarcinomas of the digestive system.

Modern Pathology (2008) 21, 1379-1386; doi:10.1038/modpathol.2008.107; published online 13 June 2008
\end{abstract}

Keywords: cadherin-17; immunohistochemistry; adenocarcinoma

Cancer of an unknown primary site is the seventh to eighth most frequently occurring cancer found in clinical practice. ${ }^{1}$ The age-adjusted annual incidence per 100000 population is $7-12$ cases in the United States, 18 in Australia, and 6 in the Netherlands. It is the fourth commonest cause of cancer death in both men and women. ${ }^{2}$ Because the optimal treatment of cancer is linked to the primary tumor site, failure to identify the primary site causes considerable problems during management of patients. Adenocarcinomas account for almost half of cancers of unknown primary site. The use of immunohistochemistry to detect specific antigens in tumor cells has led to significant improvement in the diagnosis of cancers. For example, prostatespecific antigen is used as a marker of prostatic

Correspondence: Dr Y-M Jeng, MD, Department of Pathology, College of Medicine, National Taiwan University Hospital, National Taiwan University, 7, Chung-Shan South Road, Taipei, Taiwan, ROC.

E-mail: mrna0912@yahoo.com.tw

Received 28 January 2008; revised 13 May 2008; accepted 18 May 2008; published online 13 June 2008 cancer. ${ }^{3}$ Thyroid transcription factor is a marker of pulmonary cancer. ${ }^{4}$ However, a good marker for gastrointestinal (GI) tumors is still lacking.

Cadherin-17, also called liver-intestinal cadherin or human peptide transporter-1, is a member of the cadherin superfamily and is a $\mathrm{Ca}^{2+}$-dependent cell-cell adhesion molecule. ${ }^{5,6}$ Unlike the so-called classic cadherins, such as E-, N-, and P-cadherins, cadherin-17 has seven cadherin repeats instead of five within the extracellular domain and only 20 amino-acid residues in the cytoplasmic domain. The markedly short cytoplasmic domain lacks homology with other cadherins and the adhesive function of cadherin-17 is not dependent on association with other cytoplasmic proteins. ${ }^{7}$ The subcellular distribution of cadherin-17 is also different from classic cadherins. In intestinal epithelial cells, E-cadherin is concentrated in adherens junctions whereas cadherin-17 is evenly distributed along the lateral contact area. ${ }^{8}$

The tissue distribution of cadherin-17 differs by species. In the rat, cadherin-17 is expressed in the liver and intestinal epithelial cells. In human and mouse, expression is limited to intestinal epithelial 
Table 1 Cadherin-17 immunohistochemical staining in normal tissues

\begin{tabular}{ll} 
No staining & \\
Adrenal cortex & Skeletal muscle \\
Bile duct epithelium & Skin \\
Blood vessel & Smooth muscle, uterus \\
Breast (duct and lobule) & Spleen \\
Cerebrum & Stomach, antrum \\
Cerebellum & Stomach, body \\
Endometrial epithelium & Testis, Leydig cells \\
Endometrial stroma & Testis seminiferous \\
& tubules \\
Esophagus, squamous & Thymus \\
epithelium & Tonsil \\
Heart myocardium & \\
& \\
Kidney, cortex & A+ staining \\
Kidney medulla & Appendicular \\
Liver & epithelium \\
& Colonic epithelium \\
Lung & Small intestine \\
Lymph node & epithelium \\
& \\
Pancreas acinar cells & \\
Pancreas islets cells & \\
Placenta & \\
Prostate & \\
Salivary gland, parotid & \\
\hline
\end{tabular}

cells and is not found in the liver. ${ }^{9}$ The limited distribution of cadherin-17 in normal tissues suggests that it may be a useful marker for identification of primary sites of tumors. It has been reported that cadherin-17 is expressed in colorectal, ${ }^{10}$ gastric, ${ }^{11,12}$ and pancreatic cancers. ${ }^{13}$ Whether it is expressed in tumors in other organs is unknown.

Accordingly, in this study, we examined the expression of cadherin-17 in tumors derived from various body sites using tissue arrays. We found cadherin-17 was diffusely and strongly expressed in colorectal adenocarcinomas, scattered expressed in adenocarcinomas of the stomach, the pancreas and the bile duct, and virtually not expressed in tumors of other body sites. Our study indicates cadherin-17 may be a useful marker for identification of primary sites of metastatic adenocarcinomas.

\section{Materials and methods}

\section{Tissue Specimens}

Formalin-fixed, paraffin-embedded tissue blocks from 518 previously characterized primary carcinomas and 60 nontumorous tissues were obtained from the archives of the Department of Pathology, National Taiwan University Hospital. The study was conducted according to the guidelines of our institutional review board. These tumor cases included 44 breast cancers, 39 carcinomas of the uterine cervix, 33 cholangiocarcinomas, 47 colorectal cancers, 57 hepatocellular carcinomas, 50 lung cancers, 47 renal cell carcinomas, 89 ovarian

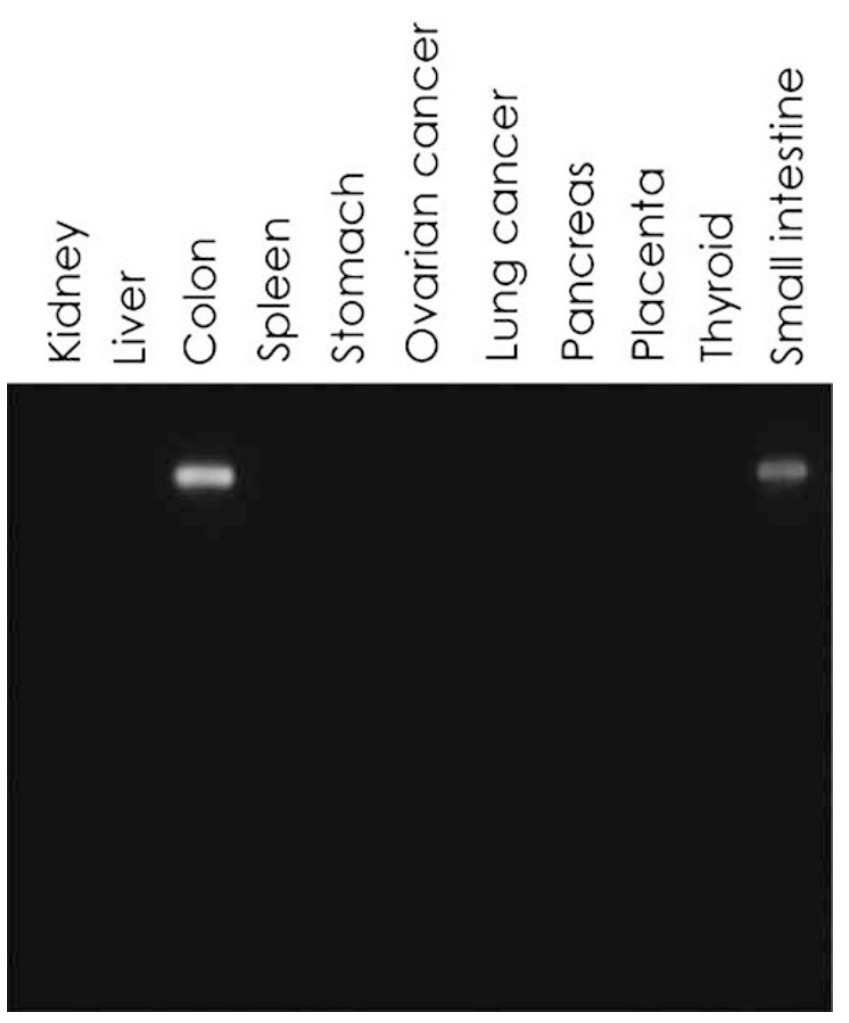

Figure 1 Western blot analysis of cadherin-17 protein in tissue lysates. Equal amounts of the protein $(25 \mu \mathrm{g})$ were loaded in each lane. Among these tumors, only colon and small intestine tissue expressed cadherin-17 protein. Cadherin-17 is seen as a single band with a molecular weight of $110 \mathrm{kDa}$.

cancers, 28 pancreatic cancers, 39 prostatic cancers, and 45 gastric cancers. The spectrum of nontumorous tissue examined is listed in Table 1.

\section{Tissue Arrays}

Targeted tissue areas were marked on H\&E-stained sections. A $2 \mathrm{~mm}$ tissue core was removed for each case using a manual tissue array device (Beecher Instruments, Silver Spring, MD, USA) and inserted into a recipient paraffin block. The arrayed tissues were cut into $4-\mu \mathrm{m}$ thick slices and placed on positively charged slides.

\section{Western Blot Analysis}

Western blots were carried out on the protein lysates of 11 representative normal and tumor tissues, including kidney, liver, colon, small intestine, stomach, spleen, thyroid, pancreas, placenta, and carcinomas from ovary and lung. Similar amounts of total protein from each lysate were separated by $8 \%$ Tris-glycine-SDS-polyacrylamide gels (Novex, San Diego, CA, USA), and then electroblotted to Millipore Immobilon-P 

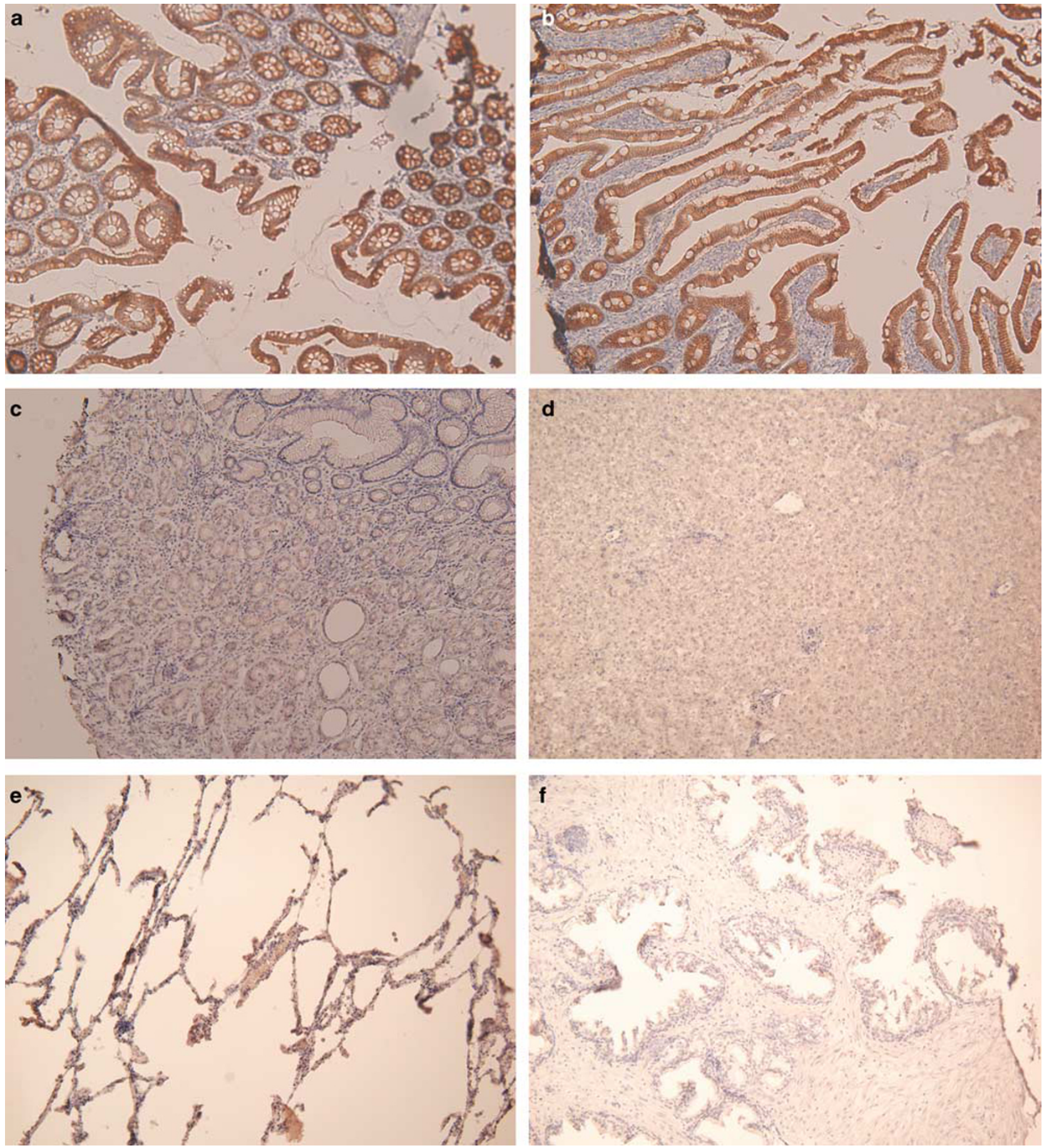

Figure 2 Immunohistochemical staining of cadherin-17 in normal tissue. Cadherin-17 was expressed in epithelial cells of colon (a) and small intestine (b), but not in gastric mucosa (c), liver (d), lung (e), or prostate (f).

polyvinylidene difluoride membranes. The membranes were probed with anti-cadherin-17 mouse monoclonal antibody, clone $1 \mathrm{H} 3$ (IgG1), at a concentration of $1 \mu \mathrm{g} / \mathrm{ml}$ (Abnova, Taipei, Taiwan) followed by a peroxidase-conjugated goat antimouse immunoglobulin (1:2500). Western blots were developed using chemiluminescence (Pierce, Rockford, IL, USA).

\section{Immunohistochemical Staining}

Tissue sections were dewaxed and rehydrated. Antigen retrieval was done by incubating slides in $0.01 \mathrm{M}$ citric acid buffer (pH 6.0) at $100^{\circ} \mathrm{C}$ for $10 \mathrm{~min}$. After blocking with $3 \% \mathrm{H}_{2} \mathrm{O}_{2}$ and $5 \%$ fetal bovine serum, slides were allowed to react with a monoclonal antibody against cadherin-17 (clone 1H3, 
Table 2 Immunohistochemical staining of cadherin-17, cytokeratin 7, cytokeratin 20, and CDX-2 in human cancers

\begin{tabular}{|c|c|c|c|c|c|c|c|c|c|c|c|c|c|c|c|c|c|c|c|c|}
\hline & \multicolumn{5}{|c|}{ Cadherin-17 } & \multicolumn{5}{|c|}{ Cytokeratin 7} & \multicolumn{5}{|c|}{ Cytokeratin 20} & \multicolumn{5}{|c|}{$C D X-2$} \\
\hline & 0 & $1+$ & $2+$ & $3+$ & $4+$ & 0 & $1+$ & $2+$ & $3+$ & $4+$ & 0 & $1+$ & $2+$ & $3+$ & $4+$ & 0 & $1+$ & $2+$ & $3+$ & $4+$ \\
\hline Breast cancer & 44 & 0 & 0 & 0 & 0 & 3 & 1 & 0 & 1 & 38 & 41 & 0 & 0 & 1 & 0 & 46 & 0 & 0 & 0 & 0 \\
\hline \multicolumn{21}{|l|}{ Cervical carcinoma } \\
\hline Adenocarcinoma & 6 & 0 & 0 & 0 & 0 & 0 & 2 & 0 & 0 & 4 & 6 & 0 & 1 & 0 & 0 & 7 & 0 & 0 & 0 & 0 \\
\hline Squamous cell carcinoma & 33 & 0 & 0 & 0 & 0 & 7 & 3 & 1 & 1 & 21 & 35 & 0 & 0 & 0 & 0 & 36 & 0 & 0 & 0 & 0 \\
\hline Cholangiocarcinoma & 24 & 5 & 0 & 1 & 3 & 0 & 1 & 2 & 2 & 24 & 25 & 2 & 2 & 0 & 7 & 30 & 3 & 1 & 1 & 1 \\
\hline Colorectal adenocarcinoma & 2 & 0 & 3 & 4 & 38 & 39 & 5 & 1 & 0 & 0 & 8 & 2 & 5 & 5 & 28 & 4 & 0 & 1 & 1 & 47 \\
\hline Hepatocellular carcinoma & 57 & 0 & 0 & 0 & 0 & 41 & 4 & 3 & 3 & 3 & 54 & 0 & 0 & 0 & 0 & 55 & 0 & 0 & 0 & 0 \\
\hline \multicolumn{21}{|l|}{ Lung } \\
\hline Adenocarcinoma & 39 & 0 & 0 & 0 & 0 & 0 & 1 & 1 & 2 & 34 & 36 & 3 & 1 & 0 & 1 & 41 & 0 & 0 & 0 & 0 \\
\hline Squamous cell carcinoma & 11 & 0 & 0 & 0 & 0 & 5 & 2 & 0 & 1 & 3 & 11 & 0 & 0 & 0 & 0 & 11 & 0 & 0 & 0 & 0 \\
\hline \multicolumn{21}{|l|}{ Kidney (renal cell carcinoma) } \\
\hline Conventional type & 42 & 0 & 0 & 0 & 0 & 32 & 5 & 0 & 1 & 2 & 32 & 5 & 1 & 1 & 0 & 41 & 0 & 0 & 0 & 0 \\
\hline Chromophobe & 1 & 0 & 0 & 0 & 0 & 0 & 0 & 0 & 0 & 0 & 0 & 0 & 0 & 0 & 1 & 1 & 0 & 0 & 0 & 0 \\
\hline Collecting duct & 1 & 0 & 0 & 0 & 0 & 0 & 0 & 0 & 0 & 0 & 1 & 0 & 0 & 0 & 0 & 1 & 0 & 0 & 0 & 0 \\
\hline Papillary & 3 & 0 & 0 & 0 & 0 & 0 & 0 & 0 & 0 & 3 & 3 & 0 & 0 & 0 & 0 & 3 & 0 & 0 & 0 & 0 \\
\hline \multicolumn{21}{|l|}{ Ovary } \\
\hline Serous carcinoma & 55 & 0 & 0 & 0 & 0 & 0 & 2 & 4 & 7 & 41 & 54 & 0 & 0 & 0 & 0 & 53 & 0 & 0 & 0 & 0 \\
\hline Mucinous carcinoma & 34 & 0 & 0 & 0 & 0 & 1 & 2 & 2 & 2 & 27 & 14 & 10 & 2 & 2 & 6 & 25 & 4 & 3 & 1 & 1 \\
\hline Pancreas (adenocarcinoma) & 12 & 7 & 5 & 3 & 1 & 0 & 1 & 1 & 1 & 23 & 15 & 9 & 1 & 0 & 0 & 25 & 1 & 0 & 0 & 2 \\
\hline Prostate (adenocarcinoma) & 38 & 0 & 0 & 0 & 1 & 25 & 3 & 2 & 1 & 0 & 32 & 5 & 1 & 1 & 0 & 35 & 0 & 0 & 0 & 0 \\
\hline Stomach adenocarcinoma & 20 & 8 & 5 & 8 & 4 & 3 & 5 & 5 & 4 & 26 & 42 & 1 & 0 & 0 & 1 & 16 & 8 & 6 & 5 & 12 \\
\hline
\end{tabular}

Abnova), cytokeratin 7 (DakoCytomation, Glostrup, Denmark), cytokeratin 20 (DakoCytomation), or CDX-2 (Biogenix, San Ramon, CA, USA) at a dilution of $1: 100$ at $4^{\circ} \mathrm{C}$ overnight. Slides were then incubated with polymer-HRP reagent (BioGenex). Peroxidase activity was visualized with diaminobenzidine tetrahydroxychloride solution (BioGenex). Sections were counterstained with hematoxylin. Dark brown membranous staining was defined as positive and no staining was defined as negative. For negative controls, we replaced the primary antibody with $5 \%$ fetal bovine serum. Staining was scored as follows: 0 (no detectable staining); $1+(<25 \%$ positive cells), $2+(25-49 \%) ; 3+$ $(50-74 \%)$; and $4+(>75 \%)$. In cells with positive staining, the staining was intense and uniform, so intensity was not factored into the scoring.

\section{Results}

We first determined the specificity of the anticadherin-17 antibody (clone 1H3) by western blot analysis. Cadherin-17 expression was detected exclusively in human lysates of small intestine and colon and not in lysates from other normal tissues and tumors (Figure 1). The antibody was specific to the cadherin-17 protein because a single band with apparent molecular weight of about $110 \mathrm{kDa}$ was present in lysates of small intestine and colon. This band corresponded to the cadherin-17 protein.
There was no detectable cross reactivity with other proteins as revealed in the western blot analysis.

Table 1 shows the distribution of cadherin-17 in normal tissues by immunohistochemistry on samples from tissue arrays. Strong diffuse membranous staining of cadherin-17 was seen in intestinal epithelium, including those samples taken from small intestine, appendix, and colorectal mucosa (Figures 2a and b). Cadherin-17 was expressed in the surface epithelium of duodenum but not in Brunner's gland. Cadherin-17 was not expressed in normal gastric mucosa (Figure 2c) but was seen in areas with intestinal metaplasia. No other tissue type had detectable cadherin-17 expression (Figures 2d-f).

Table 2 shows results of cadherin-17 immunohistochemical staining for 518 tumors from various body sites. Membranous staining of cadherin-17 was present in $45(96 \%)$ of 47 colorectal cancers; 38 (81\%) of the 47 showed diffuse staining ( $>75 \%$ ) (Figure 3a). Cadherin-17 was also expressed in $25(56 \%)$ of 45 gastric adenocarcinomas, $16(57 \%)$ of 28 pancreatic adenocarcinomas, and $9(27 \%)$ of 33 cholangiocarcinomas (Figures 3b-d). Most of the gastric, pancreatic, and biliary adenocarcinomas (42 of $50,84 \%$ ) showed a focal or scattered staining pattern. Cadherin-17 was rarely expressed in tumors of other body sites (Figures 3e and f). Of the 331 tumors examined, cadherin-17 was expressed in only $1(0.3 \%)$, a prostatic adenocarcinoma. 

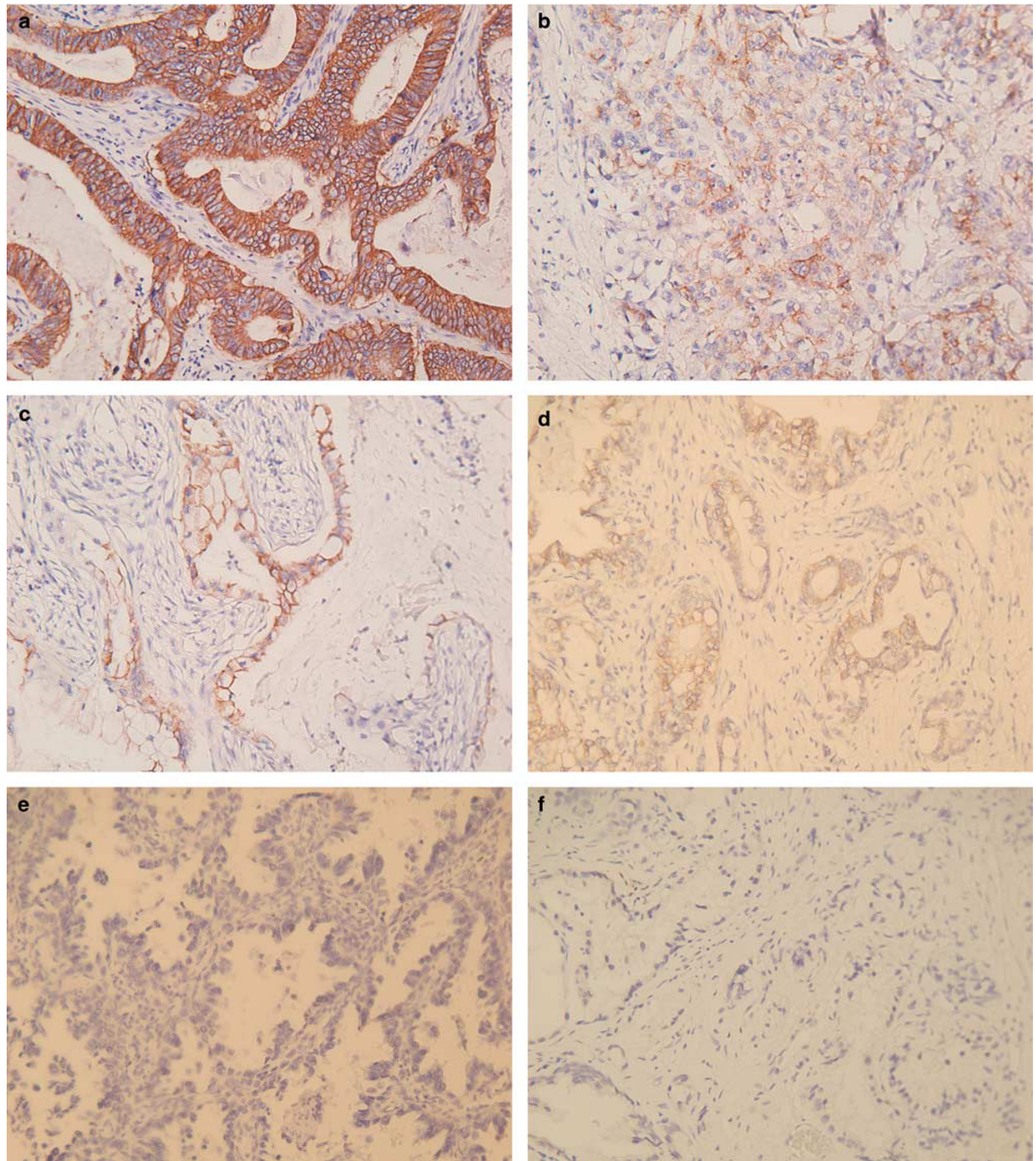

Figure 3 Immunohistochemical staining of cadherin-17 in tumor tissue. Cadherin 17 was expressed in colorectal adenocarcinoma (a), gastric adenocarcinoma (b), pancreatic ductal adenocarcinoma (c), and cholangiocarcinoma (d), but not in pulmonary (e) or prostatic adenocarcinoma (f).

To compare with current standard markers for the identification of GI adenocarcinomas, the same arrays were stained with cytokeratin 7 , cytokeratin 20, and CDX2. Similar to cadherin-17, CDX2 showed diffuse staining in colorectal carcinomas, and more scattered staining patterns in gastric, pancreatic, and biliary carcinomas. The sensitivity of cadherin-17 was slightly better than CDX2 (colorectal, 96 vs $92 \%$; gastric, 56 vs $66 \%$; pancreatic, 57 vs $11 \%$; biliary, 27 vs $16 \%$ ). CDX2 was expressed in $26 \%$ of mucinous ovarian carcinomas, a common differential diagnosis for colorectal adenocarcinoma. In 

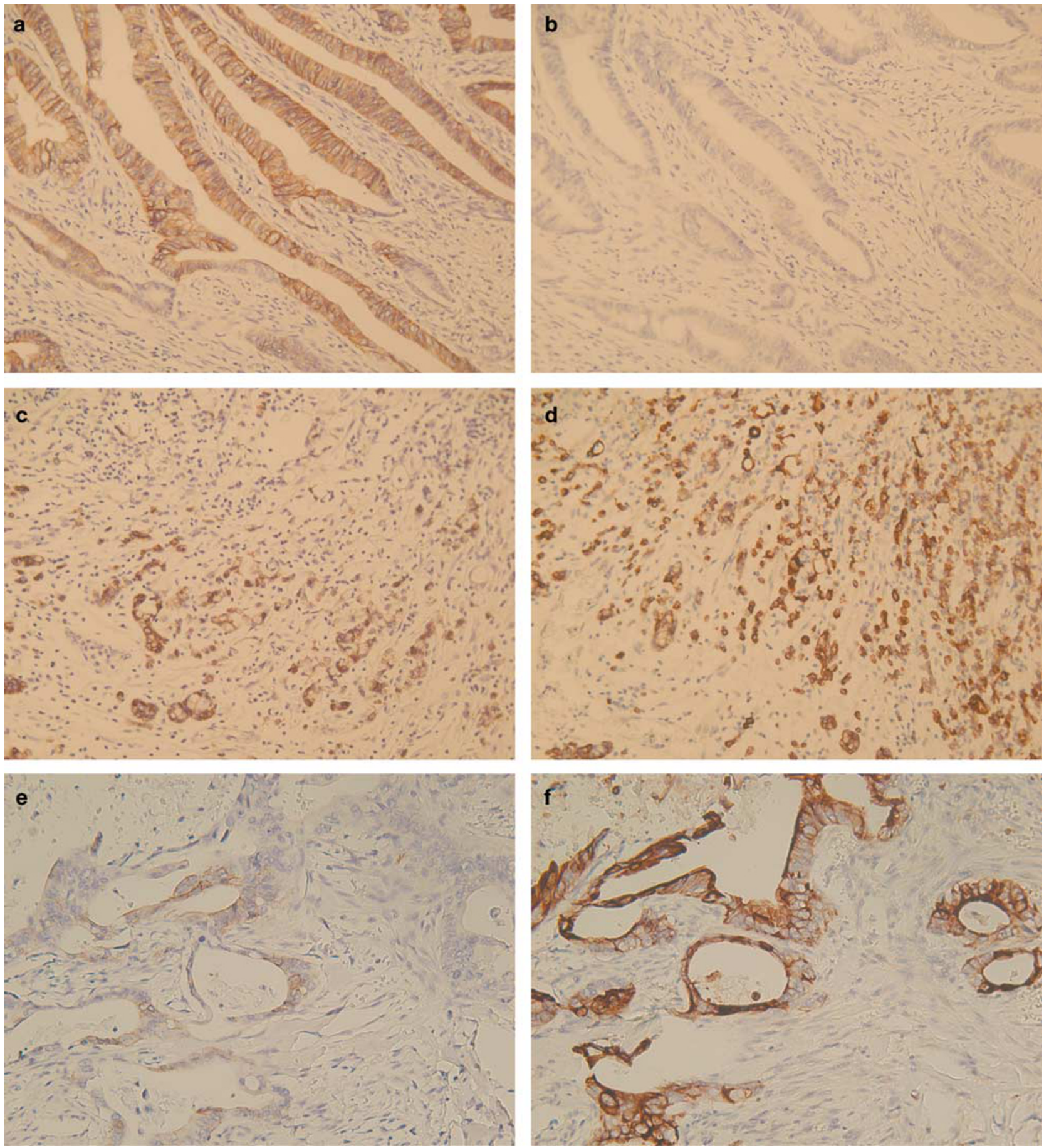

Figure 4 Expression of cadherin-17 and cytokeratin 7 in colorectal, gastric and pancreatic adenocarcinoma. (a, b) A colorectal adenocarcinoma positive for cadherin-17 (a) and negative for cytokeratin 7 (b). (c, d) A gastric adenocarcinoma positive for cadherin-17 (c) and cytokeratin 7 (d). (e, f) A pancreatic adenocarcinoma positive for cadherin-17 (e) and cytokeratin 7 (f).

contrast, none of the 34 mucinous ovarian carcinomas expressed cadherin-17. These results suggest cadherin-17 is a comparable, if not better marker, than CDX2. As in previous reports, ${ }^{14}$ we found that cytokeratin 7 is frequently expressed in adenocarcinomas from most organs but not expressed in colorectal cancers. We reasoned that a cadherin-17/ cytokeratin 7 two-marker profile may further differ- entiate colorectal cancers from those arising from upper GI organs. Immunostaining of cytokeratin 7 in our tissue array showed that 37 of $47(79 \%)$ of colorectal adenocarcinomas were cadherin-17 $(+) /$ cytokeratin $7(-)$ (Table 3a; Figures $4 \mathrm{a}$ and b). Another $6(13 \%)$ colorectal adenocarcinomas were cadherin-17(+)/cytokeratin $7(+)$. In total, 24 of 45 $(53 \%)$ gastric adenocarcinomas, 16 of $28(51.7 \%)$ 
a Colorectal cancer
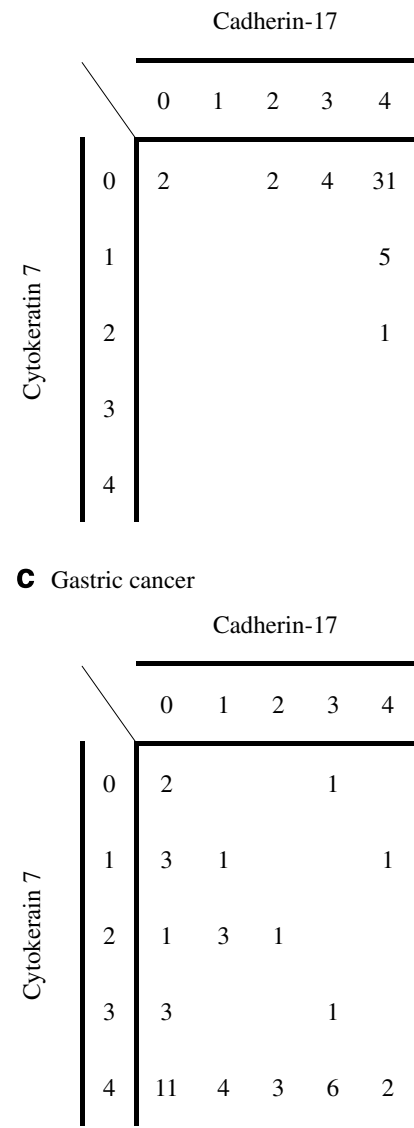

b Pancreatic cancer

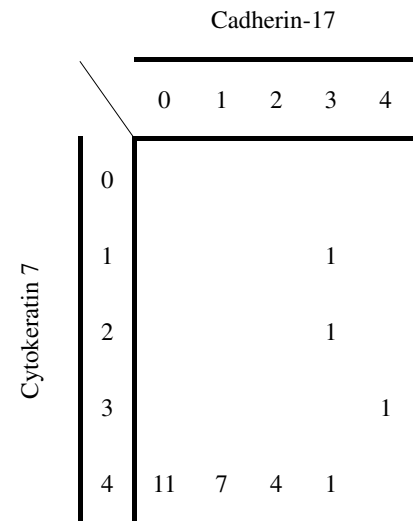

d Cholangiocarcinoma

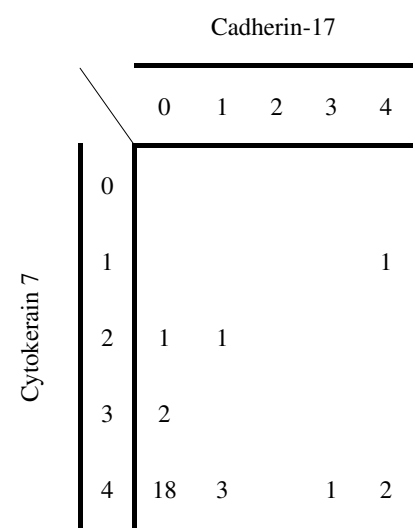

pancreatic adenocarcinomas, and $9(27 \%)$ of 33 cholangiocarcinomas were cadherin-17 $(+)$ / cytokeratin $7(+)$ (Figures 4c-f). One gastric adenocarcinoma was cadherin-17(+)/cytokeratin $7(-)$ (Table $3 b-d)$. The staining of cytokeratin 20 in tumors was heterogeneous and did not give information additional to that obtained from the other three markers.

\section{Discussion}

Adenocarcinoma of an unknown primary site is one of the most common clinical problems. The seven commonest primary sites are breast, colon, lung, ovary, pancreas, prostate, and stomach. ${ }^{15}$ Because metastatic adenocarcinomas from different locations may have a similar microscopic appearance, identification of the primary site is difficult. A number of immunohistochemical markers were identified to assist the histologic diagnosis such as cytokeratin 7 , cytokeratin 20, thyroid transcription factor 1, CDX2, prostate-specific antigen, and mesothelin. ${ }^{3,4,15}$ However, a highly specific marker for GI adenocarcinoma is still lacking.
Cadherin-17 is an adhesion protein that is normally expressed only in intestinal epithelium. This highly specific localization makes cadherin-17 a potential tool for immunohistochemical diagnosis. Our results show that cadherin-17 expression is very common in colorectal adenocarcinomas and is also expressed in more than half of gastric and pancreatic adenocarcinomas, and in a small number of cholangiocarcinomas. Its expression is not limited to tumors with intestinal metaplasia. Furthermore, expression of cadherin-17 is very uncommon in carcinomas outside the GI tract. These observations make cadherin-17 a useful marker for histologic diagnosis of adenocarcinoma of unknown primary site.

To further differentiate tumors from the colorectal region from those derived from the upper GI tract, two approaches were used. Expression of cadherin17 in colorectal adenocarcinoma was usually diffuse and strong whereas expression in adenocarcinomas of the upper GI tract was usually focal or scattered. The other approach we used was immunostaining of cytokeratin 7. Immunoreactivity of cytokeratin 7 was seen in adenocarcinomas from most body sites, which limits its clinical application. However, using cadherin-17/cytokeratin 7 two-marker profiles 
greatly narrowed down the candidate primary site. In our data set, 37 of $38(97.3 \%)$ cadherin- $17(+) /$ cytokeratin $7(-)$ tumors were colorectal adenocarcinomas. The other one was a gastric adenocarcinoma. Of 56, $49(86.0 \%)$ cadherin-17(+)/cytokeratin $7(+)$ tumors were gastric, pancreatic, or biliary adenocarcinomas, 6 were colorectal adenocarcinomas, and 1 was a prostatic adenocarcinoma. Hence, the cadherin-17/cytokeratin 7 profile can differentiate adenocarcinoma of upper and lower GI tract with high specificity.

In our tissue array, cadherin-17 was expressed in more than $95 \%$ of colorectal adenocarcinomas. One of the two cases immunonegative for cadherin-17 fulfilled the criteria of large cell minimally differentiated carcinomas of the colon, ${ }^{16}$ a tumor characterized by loss of glandular differentiation and microsatellite instability. The other was an adenosquamous carcinoma. Cadherin-17 was only expressed in slightly over half of gastric and pancreatic adenocarcinomas and $27 \%$ of cholangiocarcinomas. These results indicate a cadherin-17(-) adenocarcinoma is very unlikely to be a colorectal adenocarcinoma, but the possibility of it arising from stomach, pancreas, and biliary tract cannot be completely ruled out.

The tissue samples used in our tissue array were obtained from the primary sites of the tumor. Whether cadherin-17 retained its utility in metastatic tumors remains to be clarified. In gastric cancer, cadherin-17 expression was significantly more frequent in advanced-stage cases, ${ }^{11}$ but in pancreatic and colorectal cancer, cadherin-17 was more frequent in well-differentiated tumors ${ }^{10,13}$ without lymph node metastasis. Further testing using biopsy specimens from additional metastatic cancers would be worthwhile.

\section{Acknowledgement}

This work was supported by grants from Min-Sheng General Hospital (to M-C Su) and National Taiwan University Hospital (to Y-M Jeng).

\section{References}

1 Pavlidis N. Forty years experience of treating cancer of unknown primary. Acta Oncol 2007;46:592-601.
2 Levi F, Te VC, Erler G, et al. Epidemiology of unknown primary tumours. Eur J Cancer 2002;38:1810-1812.

3 Nadji M, Tabei SZ, Castro A, et al. Prostatic-specific antigen: an immunohistologic marker for prostatic neoplasms. Cancer 1981;48:1229-1232.

4 Fabbro D, Di Loreto C, Stamerra O, et al. TTF-1 gene expression in human lung tumours. Eur J Cancer 1996;32A:512-517.

5 Berndorff D, Gessner R, Kreft B, et al. Liver-intestine cadherin: molecular cloning and characterization of a novel $\mathrm{Ca}^{++}$-dependent cell adhesion molecule expressed in liver and intestine. J Cell Biol 1994;125: 1353-1369.

6 Dantzig AH, Hoskins JA, Tabas LB, et al. Association of intestinal peptide transport with a protein related to the cadherin superfamily. Science 1994;264: 430-433.

7 Kreft B, Berndorff D, Böttinger A, et al. LI-cadherinmediated cell-cell adhesion does not require cytoplasmic interactions. J Cell Biol 1997;136:1109-1121.

8 Wendeler MW, Drenckhahn D, Gessner R, et al. Intestinal LI-cadherin acts as a Ca2+-dependent adhesion switch. J Mol Biol 2007;370:220-230.

9 Gessner R, Tauber R. Intestinal cell adhesion molecules. Liver-intestine cadherin. Ann NY Acad Sci 2000;915:136-143.

10 Takamura M, Ichida T, Matsuda Y, et al. Reduced expression of liver-intestine cadherin is associated with progression and lymph node metastasis of human colorectal carcinoma. Cancer Lett 2004;212:253-259.

11 Ito $\mathrm{R}$, Oue $\mathrm{N}$, Yoshida $\mathrm{K}$, et al. Clinicopathological significant and prognostic influence of cadherin-17 expression in gastric cancer. Virchows Arch 2005;447:717-722.

12 Ko S, Chu KM, Luk JM, et al. CDX2 co-localizes with liver-intestine cadherin in intestinal metaplasia and adenocarcinoma of the stomach. J Pathol 2005;205: 615-622.

13 Takamura M, Sakamoto M, Ino Y, et al. Expression of liver-intestine cadherin and its possible interaction with galectin-3 in ductal adenocarcinoma of the pancreas. Cancer Sci 2003;94:425-430.

14 Dennis JL, Hvidsten TR, Wit EC, et al. Markers of adenocarcinoma characteristic of the site of origin: development of a diagnostic algorithm. Clin Cancer Res 2005;11:3766-3772.

15 Dennis JL, Vass JK, Wit EC, et al. Identification from public data of molecular markers of adenocarcinoma characteristic of the site of origin. Cancer Res 2002;62: 5999-6005.

16 Hinoi T, Tani M, Lucas PC, et al. Loss of CDX2 expression and microsatellite instability are prominent features of large cell minimally differentiated carcinomas of the colon. Am J Pathol 2001;159: 2239-2248. 